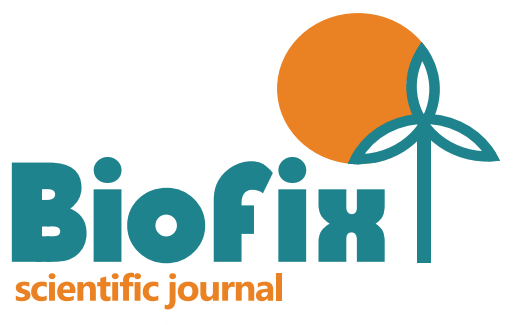

v. 2 n. 22017

\section{INFLUÊNCIA DAS CONDIÇÕES AMBIENTAIS NO CRESCIMENTO DE DOIS GRUPOS ARBÓREOS EM FLORESTA COM ARAUCÁRIA}

\author{
INFLUENCE OF ENVIRONMENTAL CONDITIONS ON THE GROWTH OF
} TWO TREE GROUPS IN ARAUCARIA FOREST
Recebido em 01/07/2017

Aceito em 21/07/2017

Publicado em 10/08/2017

DOI: dx.doi.org/10.5380/biofix.v2i2.53634

\author{
Simone Filipini Abrão ${ }^{1}$ \\ Nivaldo Eduardo Rizzi \\ Allan Libanio Pelissari ${ }^{3}$ \\ Sebastião do Amaral Machado ${ }^{4}$
}

\begin{abstract}
Universidade Federal do Paraná, Curitiba, Paraná, Brasil simone.abrao@ufpr.br' ${ }^{1}$,niva@ufpr.br ${ }^{2}$,allanpelissari@gmail.com ${ }^{3}$ \& samachado@ufpr.br ${ }^{3}$
\end{abstract}

\section{RESUMO}

O crescimento da vegetação está relacionado com a disponibilidade de recursos naturais e a capacidade de cada espécie em utilizá-los. Por isso, o objetivo deste estudo foi avaliar o crescimento de dois grupos arbóreos: Grupo 1, correspondente a espécie Araucaria angustifolia; e Grupo 2, representado pelas dez espécies com maior valor de importância em uma Floresta Ombrófila Mista. Para isso, as seguintes hipóteses foram formuladas: a espécie Araucaria angustifolia apresenta crescimento diferenciado das espécies mais representativas em função de características ambientais em que está localizada? e quais locais podem ser prioritários para o manejo dessa espécie? Este estudo foi realizado na Floresta Nacional de São Francisco de Paula, estado do Rio Grande do Sul, Brasil, onde todas as espécies com diâmetro à altura do peito $\geq 9,5 \mathrm{~cm}$ foram avaliadas por meio de inventário florestal contínuo em cinco unidades de estudo (UEs I, II, III, IV e V) por um período de dez anos. Foram calculados os incrementos correntes anuais em diâmetro para cada árvore, sendo posteriormente calculados os valores de incremento corrente médio por espécie. No último ano, foram medidas as alturas totais e comerciais. O Grupo 1 manteve crescimento superior $\left(0,32 \mathrm{~cm} \cdot \mathrm{ano}^{-1}\right)$ ao Grupo $2\left(0,23 \mathrm{~cm} \cdot \mathrm{ano}^{-1}\right)$, atribuído à capacidade de resiliência de Araucaria angustifolia. Essa espécie também demonstrou maiores alturas totais e comerciais médias, com 18,54 e 14,92 m, respectivamente, resultado que corroborou para evidenciar o seu potencial para o manejo. As UEs II, III e IV apresentaram características edáficas que podem estar relacionadas ao maior potencial de crescimento de Araucaria angustifolia, o que confere a espécie um crescimento diferenciado ao das demais espécies da floresta.

PALAVRAS-CHAVE: Araucaria angustifolia, Incremento, Características edáficas.

\section{ABSTRACT}

Vegetation growth its related with the availability of natural resources and the ability of each species to use them. Therefore, the aim of this study was to evaluate the growth of two tree groups: Group 1, correspondent to Araucaria angustifolia; and Group 2, represented by the ten most important species in an Araucaria Forest. Thus, the following hypotheses were formulated: Does Araucaria angustifolia present differentiated growth of the most representative species according to the environmental conditions in which it is located? And, which sites may be priority for the management of this species? This study was carried out in the National Forest of São Francisco de Paula, RS, Brazil, where the all tree species with diameter at breast height $\geq 9.5 \mathrm{~cm}$ were evaluated by means of a continuous forest inventory in five study units (SU I, II, III, IV, and V) for a period of ten years. The current annual increment in diameter were calculated for each tree, and the mean incremental values per species were then calculated. In the last year, total and commercial heights were measured. Group 1 maintained a superior growth $\left(0.32 \mathrm{~cm} \cdot\right.$ year $\left.^{-1}\right)$ to Group $2\left(0.23 \mathrm{~cm} \cdot\right.$ year $\left.^{-1}\right)$ attributed to the resilience capacity of Araucaria angustifolia. This species also showed highest average total and commercial heights, with 18.54 and $14.92 \mathrm{~m}$, respectively, a result that corroborated to show its potential for management. SU II, III, and IV presented edaphic characteristics related to a greatest growth potential of $A r-$ aucaria angustifolia, which confers the species a differentiated growth when compared to other forest species. KEYWORDS: Araucaria angustifolia, Increment, Edaphic
characteristics. 


\section{INTRODUÇÃO}

O bioma Mata Atlântica abrange áreas do Norte ao Sul do país, em que, desde o início da colonização europeia, sofreu com o avanço sobre seus limites e com intenso extrativismo, tornando-se o bioma mais ameaçado do país, remanescendo apenas $8 \%$ de sua área original de 1.306.421 km² (SCHÄFFER; PROCHNOW, 2002). Mesmo com sua intensa fragmentação, esse bioma é considerado um dos cinco mais importantes hotspots de biodiversidade do mundo (MYERS et al., 2000).

No bioma Mata Atlântica, estão inseridos um conjunto de ecossistemas associados com grande número de endemismos (BRASIL, 2006), como, por exemplo, a Floresta com Araucária, caracterizada pela presença da conífera brasileira de maior expressão, Araucaria angustifolia (Bertol.) Kuntze. Embora a vegetação brasileira seja dominada por espécies tropicais, há resquícios de vegetações tropicais e austrais na Floresta com Araucária, resultantes das flutuações climáticas entre períodos glaciais e interglaciais que configuram a vegetação atual (LEITE; KLEIN, 1990; BACKES; IRGANG, 2004).

Iriarte e Behling (2007) ressalvam que, além do favorecimento climático, a expansão da floresta no Holoceno pode ter sido favorecida pela ação antrópica, em que registros palinológicos e arqueológicos indicam uma forte associação entre a colonização e o desenvolvimento dos povos tradicionais com o avanço da Floresta com Araucária. Para esses autores, o motivo da ocupação indígena mais intensa e permanente foi justificado pela disponibilidade de recursos, principalmente pela presença da semente de Araucaria angustifolia.

Durante grande parte do século $X X$, a exploração de Araucaria angustifolia foi a principal fonte madeireira do país (KOCH; CORRÊA, 2010), em que, em muitas ocasiões, o desmatamento foi estimulado pelo próprio governo (SCHÄFFER; PROCHNOW, 2002). Devido a exploração predatória, a Floresta com Araucária tornou-se um dos ecossistemas mais ameaçados (CARLUCCI et al., 2011).

Diante da demanda por produtos florestais madeireiros e não madeireiros oriundos de florestas homogêneas ou heterogêneas, é necessário avaliar o potencial produtivo para garantir a sustentabilidade. Portanto, medidas que possibilitem sua exploração sustentável, amparada pela legislação e com o respeito ao meio ambiente devem ser desenvolvidos e praticados.

Este trabalho tem como objetivo avaliar o crescimento de dois grupos arbóreos, um com a espécie Araucaria angustifolia e outro com as dez espécies de maior valor de importância em um remanescente de Floresta Ombrófila
Mista, para responder as seguintes hipóteses: a espécie Araucaria angustifolia apresenta crescimento diferenciado das espécies mais representativas em função do ambiente em que está localizada? Quais locais podem ser prioritários para o manejo dessa espécie?

\section{MATERIAL E MÉTODOS}

Este estudo foi conduzido na Floresta Nacional de São Francisco de Paula, localizada no município de São Francisco de Paula, região nordeste do estado do Rio Grande do Sul, Brasil, entre as coordenadas geográficas $29^{\circ} 25^{\prime} 24^{\prime \prime}$ de latitude Sul e 50²3'13" de longitude Oeste.

O clima predominante na região é do tipo $\mathrm{Cfb}$, temperado úmido, de acordo com a classificação climática de Köppen, em que a temperatura média mensal é de $14,1^{\circ} \mathrm{C}$, sendo inferior a $22^{\circ} \mathrm{C}$ no mês mais quente e superior a $3^{\circ} \mathrm{C}$ no mês mais frio. A precipitação média anual é de $2.468 \mathrm{~mm}$, distribuída de forma regular durante o ano (MORENO, 1961; MALUF, 2000) e com incidência de chuvas de até $205 \mathrm{~mm}$ em apenas 24 horas (BRASIL, 1973). A altitude local varia entre 630 a $930 \mathrm{~m}$ acima do nível do mar. O relevo apresenta-se de ondulado a fortemente ondulado. O solo predominante é o Cambissolo Húmico Alumínico típico (EMBRAPA, 2013).

A vegetação local, com diâmetro à altura do peito (DAP) igual ou superior a $9,5 \mathrm{~cm}$ foi avaliada por meio de inventário florestal contínuo, ao passo que cinco unidades de estudo (UEs I, II, III, IV e V) de um hectare cada (100 $\mathrm{m} \mathrm{x}$ $100 \mathrm{~m}$ ) foram avaliadas por um período de dez anos (2000 até 2009).

Foram calculados os parâmetros fitossociológicos relacionados à estrutura horizontal (densidade, frequência, dominância e seus respectivos valores relativos, valor de cobertura e valor de importância) para cada UE e separadas em dois grupos, um contendo a espécie Araucaria angustifolia (Grupo 1) e outro grupo formado pelas dez espécies com maior valor de importância em cada UE (Grupo 2). Por meio das medições contínuas dos diâmetros, foram calculados os incrementos correntes anuais em diâmetro (ICA) para cada árvore, sendo determinados, a partir desses, os valores de incremento corrente médio por espécie.

Para comparar o crescimento dos grupos, as UEs foram alocadas em locais diferenciados, caracterizados por critérios de densidade de Araucaria angustifolia (uma UE em local com maior densidade, uma UE em local de menor densidade, e três UEs em locais com densidade intermediária), visando contemplar a heterogeneidade dos componentes abióticos (Tabela 1). 
Tabela 1. Descrição das características ambientais das unidades de estudo em remanescente de Floresta Ombrófila Mista na Floresta Nacional de São Francisco de Paula, RS, Brasil

\begin{tabular}{|c|c|}
\hline UE & Características bióticas e abióticas \\
\hline $\mathbf{I}$ & $\begin{array}{l}\text { Seletividade higrófila, com predominância da espécie Sebastiania commersoniana (Baill.) L.B. Sm. \& Downs; Neossolo Litólico } \\
\text { apresentando restrições físico-hídricas devido a sua baixa profundidade efetiva (0,20 m); camadas de solo rico em carbono } \\
\text { orgânico; solo com estrutura granular e textura média a argilosa; sítio com baixo gradiente de declividade (10\%); espécies com } \\
\text { maior valor de importância: Ilex brevicuspis Reissek, Blepharocalyx salicifolius (Kunth) O. Berg, Sebastiania commersoniana, } \\
\text { Eugenia uruguayensis Cambess., Nectandra megapotamica Spreng. Mez, Siphoneugena reitzii D. Legrand, Cinnamomum glaziovii } \\
\text { (Mez) Kosterm., Casearia decandra Jacq., Cryptocarya aschersoniana Mez e Ocotea pulchella Mart.; a espécie Araucaria } \\
\text { angustifolia ocupou a décima oitava posição em valor de importância na UE. }\end{array}$ \\
\hline II & $\begin{array}{l}\text { Predominância da espécie Siphoneugena reitzii; profundidade mínima do solo de } 0,60 \mathrm{~m} \text {, com presença de fragmentos de rocha } \\
\text { basáltica na profundidade de } 0,45 \mathrm{~m} \text {, atestando o baixo grau de alteração do material de origem; solo com estrutura granular e } \\
\text { textura argilosa; camadas superficiais ricas em carbono orgânico; sítio com gradiente de declividade de } 24 \% \text {; espécies com maior } \\
\text { valor de importância: Siphoneugena reitzii, Podocarpus lambertii Klotzsch ex Endl., Ilex brevicuspis, Vernonanthura discolor } \\
\text { (Spreng.) H. Rob., Lamanonia ternata Vell, Blepharocalyx salicifolius, Casearia decandra, Myrsine umbellata Mart., Eugenia } \\
\text { uruguayensis Cambess e Lithraea brasiliensis Marchand; a espécie Araucaria angustifolia ocupou a quarta posição em valor de } \\
\text { importância na UE. }\end{array}$ \\
\hline III & $\begin{array}{l}\text { Profundidade mínima do solo de 0,60 m, com estrutura em blocos angulares a subangulares, textura argilosa a muito argilosa e } \\
\text { cores brunadas; sítio com gradiente de declividade de } 16 \% \text {; espécies com maior valor de importância: Blepharocalyx salicifolius, } \\
\text { Sebastiania brasiliensis Spreng, Matayba elaeagnoides Radlk., Ilex paraguariensis A. St.-Hil, Cryptocarya aschersoniana Mez, } \\
\text { Nectandra megapotamica (Spreng.) Mez, Casearia decandra, Campomanesia rhombea O. Berg, Dicksonia sellowiana Hook. e } \\
\text { Ocotea pulchella Mart; a espécie Araucaria angustifolia ocupou a primeira posição em valor de importância na UE. }\end{array}$ \\
\hline IV & $\begin{array}{l}\text { Profundidade mínima do solo de } 0,60 \mathrm{~m} \text {, com estrutura granular a blocos subangulares, textura média }(0,05 \mathrm{~m}) \text { a argilosa nas } \\
\text { demais camadas e cores brunadas, concreções óxido de Fe; camadas superficiais ricas em carbono orgânico; sítio com gradiente } \\
\text { de declividade de } 30 \% \text {; espécies com maior valor de importância: Ocotea puberula (Rich.) Nees, Casearia decandra, Ilex } \\
\text { paraguariensis, Blepharocalyx salicifolius, Sebastiania brasiliensis, Nectandra megapotamica, Sapium glandulosum (L.) Morong, } \\
\text { Campomanesia rhombea, Cryptocarya aschersoniana e Inga vera Kunth; a espécie Araucaria angustifolia ocupou a primeira } \\
\text { posição em valor de importância na UE. }\end{array}$ \\
\hline $\mathbf{v}$ & $\begin{array}{l}\text { Monodominância de Araucaria angustifolia; profundidade mínima do solo de 0,60 m, textura argilosa a muito argilosa, estrutura } \\
\text { granular }(0,05 \mathrm{~m}) \text {, demais camadas em blocos angulares a subangulares e cores brunadas; sítio com maior gradiente de } \\
\text { declividade }(42,5 \%) \text {; espécies com maior valor de importância: Casearia decandra, Ilex paraguariensis, Lonchocarpus campestris } \\
\text { Mart. ex Benth., Nectandra megapotamica, Ocotea puberula, Cinnamomum glaziovii, Matayba elaeagnoides Radlk., Inga vera, } \\
\text { Scutia buxifolia Reissek e Blepharocalyx salicifolius; a espécie Araucaria angustifolia ocupou a primeira posição em valor de } \\
\text { importância na UE. }\end{array}$ \\
\hline
\end{tabular}

A partir das médias de incremento, foi aplicada a análise de variância, em que cada UE consistiu em um bloco, ao passo que os grupos formados por Araucaria angustifolia e pelas espécies com maior valor de importância por UE (Grupos 1 e 2) representaram os tratamentos. A medição contínua dos diâmetros possibilitou que cada remedição anual correspondesse a uma repetição, totalizando nove repetições.

As hipóteses testadas pela análise de variância foram: nulidade $\left(\mathrm{H}_{0}\right)$, em que não há diferença entre o crescimento diamétrico dos grupos arbóreos; e entre o crescimento de cada grupo nas UEs. Caso rejeitada a hipótese de nulidade, para diferenciar as médias, o teste de Tukey foi aplicado ao nível de $5 \%$ de significância.

As alturas totais e comercias foram medidas no último ano inventariado, sendo tomadas, respectivamente, até o ponto mais alto da árvore (altura total) e até a inserção do galho mais grosso no fuste (altura comercial). A partir das alturas medidas, foram calculadas as médias para os Grupos 1 e 2 em cada UE e aplicada a análise de variância, considerando um delineamento de blocos ao acaso. As hipóteses de nulidade $\left(\mathrm{H}_{0}\right)$ testadas, considerando as alturas, foram: não há diferenças estatísticas entre as alturas nas diferentes UEs; e não há diferenças estatísticas entre as alturas dos grupos arbóreos.

\section{RESULTADOS E DISCUSSÃO}

As médias dos incrementos correntes em diâmetro (ICA) apresentaram grande variação entre as unidades de estudo (UEs), com valores entre 0,0022 cm para Araucaria angustifolia na segunda medição da UE I, até $1,0589 \mathrm{~cm}$ 
para a mesma espécie na terceira medição da UE IV. O menor incremento das espécies com maiores valores de importância também foi observado na UE I $(0,0322 \mathrm{~cm})$, quinta medição, ao passo que o maior valor encontrado ocorreu na UE III, segunda medição, representou metade do maior incremento encontrado para Araucaria angustifolia $(0,5169 \mathrm{~cm})$.

Por meio da análise de variâncias, foi rejeitada a hipótese $\mathrm{H}_{0}$, indicando que os ICA diferem estatisticamente entre os grupos arbóreos e entre as UEs (Tabela 2). As médias dos incrementos entre os grupos arbóreos diferiram pelo teste de Tukey ao nível de $5 \%$ de probabilidade, em que as médias dos incrementos de Araucaria angustifolia $\left(0,3186 \mathrm{~cm} \cdot\right.$ ano $\left.^{-1}\right)$ foram superiores à dos incrementos das espécies com maiores valores de importância $\left(0,2341 \mathrm{~cm} \cdot a^{-1}\right)$. Esse resultado é superior ao comumente encontrado, pois geralmente as coníferas são responsáveis por cerca de $40 \%$ da área basal da floresta (ENRIGHT, 2001).

Tabela 2. Análise de variância aplicada aos grupos arbóreos nas Unidades de Estudo (UEs) em remanescente de Floresta Ombrófila Mista na Floresta Nacional de São Francisco de Paula, RS, Brasil

\begin{tabular}{ccccc}
\hline $\begin{array}{c}\text { Fonte de } \\
\text { Variação }\end{array}$ & $\begin{array}{c}\text { Graus de } \\
\text { Liberdade }\end{array}$ & $\begin{array}{c}\text { Soma de } \\
\text { Quadrados }\end{array}$ & $\begin{array}{c}\text { Quadrados } \\
\text { médio }\end{array}$ & $\begin{array}{c}\text { F } \\
\text { calculado }\end{array}$ \\
\hline Grupos & 1 & 0,16068 & 0,16068 & $10,870^{* *}$ \\
UEs & 4 & 1,32203 & 0,33051 & $22,359^{* *}$ \\
Grupos x UEs & 4 & 0,23599 & 0,05900 & $3,991^{* *}$ \\
Resíduos & 80 & 1,18253 & 0,01478 & \\
\hline \multicolumn{5}{r}{ Total } \\
** significativo ao nível de 1\%; F crítico (Grupos; Grupos x UEs) = 6,964; \\
e F crítico (UEs) = 3,564
\end{tabular}

O incremento médio de Araucaria angustifolia apresentou valor superior ao observado por Figueiredo Filho et al. (2003), os quais encontraram um valor igual a $0,129 \mathrm{~cm} \cdot \mathrm{ano}^{-1} \mathrm{em}$ remanescente de Floresta Ombrófila Mista no estado do Paraná. Porém, Mattos et al. (2007) verificaram valores de incremento inseridos no intervalo de $0,11 \mathrm{~cm}$ até $1,15 \mathrm{~cm}$, com média igual a $0,40 \mathrm{~cm}$. No Paraná, Figueiredo Filho et al. (2010), avaliando o incremento médio das espécies arbóreas da Floresta com Araucária encontraram valor igual a $0,24 \mathrm{~cm} \cdot \mathrm{ano}^{-1}$, semelhante ao do presente estudo.

As médias entre as UEs apresentaram diferenças pelo teste de Tukey, em que a UE I $\left(0,0521 \mathrm{~cm} \cdot \mathrm{ano}^{-1}\right)$ diferiu das demais, devido ao baixo valor de incremento. As médias das UEs II $\left(0,3401 \mathrm{~cm} \cdot \mathrm{ano}^{-1}\right)$, III $\left(0,3682 \mathrm{~cm} \cdot \mathrm{ano}^{-1}\right)$ e IV $\left(0,3793 \mathrm{~cm} \cdot \mathrm{ano}^{-1}\right)$ não diferiram estatisticamente, sendo os maiores diâmetros médios encontrados na UE IV. A UE V apresentou valores intermediários de incremento $(0,2386$ $\left.\mathrm{cm} \cdot \mathrm{ano}^{-1}\right)$, não diferindo da UE II pelo teste de Tukey.

A interação entre os Grupos e as UEs foi altamente significativa, o que indica o sinergismo entre o incremento dos grupos com as UEs. Portanto, para as UEs que apresentam um maior potencial de incremento diamétrico, maior será o potencial de crescimento de Araucaria angustifolia e das espécies com maior importância na floresta.

As alturas total e comercial da UE III dos Grupos arbóreos 1 e 2 foram aproximadamente de 1,58 e 2,75 vezes maiores quando comparada às médias das alturas das demais UEs (Tabela 3). As alturas totais e comerciais médias não diferiram entre as UEs (blocos), no entanto, houve diferença estatística entre as médias dos Grupos 1 e 2 (Tabela 4). O Grupo 1, com a espécie Araucaria angustifolia, apresentou as maiores médias, sendo de $18,54 \mathrm{~m}$ e $14,92 \mathrm{~m}$, respectivamente para alturas total e comercial, ao passo que o Grupo 2 resultou nos valores de $13,96 \mathrm{~m}$ e $6,74 \mathrm{~m}$.

Tabela 3. Alturas total e comercial nas Unidades de Estudo (UEs) para os grupos arbóreos (G1 e G2) em remanescente de Floresta Ombrófila Mista na Floresta Nacional de São Francisco de Paula, RS, Brasil

\begin{tabular}{cccc}
\hline $\begin{array}{c}\text { Unidade de } \\
\text { Estudo }\end{array}$ & Grupo & $\begin{array}{c}\text { Altura total } \\
(\mathbf{m})\end{array}$ & $\begin{array}{c}\text { Altura comercial } \\
(\mathbf{m})\end{array}$ \\
\hline I & $\mathbf{1}$ & $\mathbf{1 5 , 0}$ & $\mathbf{1 1 , 7}$ \\
II & 2 & 14,9 & 6,4 \\
III & 1 & 17,8 & 14,9 \\
IV & 2 & 13,1 & 6,2 \\
& 1 & 21,5 & 17,9 \\
V & 2 & 13,6 & 6,5 \\
& 1 & 20,2 & 14,7 \\
& 2 & 14,7 & 7,6 \\
\hline
\end{tabular}

Esse resultado demonstrou o comportamento característico da espécie, pois quando Araucaria angustifolia encontra boas condições de sítio em relação à disponibilidade de recursos para se desenvolver, sobrepõem-se em relação às demais espécies, principalmente em relação a dominância e ao hábito emergente. É uma espécie que apresenta um aspecto original e contrastante com as demais árvores do Sul do Brasil, com 10 a $35 \mathrm{~m}$ de altura e 50 a $120 \mathrm{~cm}$ de diâmetro à altura do peito (CARVALHO, 2003), cujas árvores adultas normalmente formam um estrato contínuo acima de $30 \mathrm{~m}$ (RODERJAN et al., 2002). 
Tabela 4. Análise de variância aplicada as alturas totais e comerciais dos grupos arbóreos nas Unidades de Estudo (UEs) em remanescente de Floresta Ombrófila Mista na Floresta Nacional de São Francisco de Paula, RS, Brasil

\begin{tabular}{ccccc}
\hline Fonte de Variação & Graus de Liberdade & Soma de Quadrados & Quadrado Médio & F calculado \\
\hline & & Altura total & \\
\hline Grupos & 1 & 52,44100 & 52,44100 & $13,1234^{*}$ \\
UEs & 4 & 11,24000 & 2,81000 & $0,7032^{\mathrm{ns}}$ \\
Resíduos & 4 & 15,98400 & 3,99600 & \\
Total & 9 & 79,66500 & & $66,4209^{* *}$ \\
\hline & & Altura comercial & $1,0647^{\mathrm{ns}}$ \\
Grupos & 1 & 167,28100 & 2,68150 & 2,51850 \\
UEs & 4 & 10,72600 & 10,07400 & 188,08100 \\
\hline
\end{tabular}

** significativo ao nível de $1 \%$ de probabilidade; * significativo ao nível de $5 \%$ de probabilidade; e ns não significativo.

Portanto, além das características tecnológicas da madeira de Araucaria angustifolia, que favorecem seu uso no setor florestal, o incremento diamétrico e as alturas dessa espécie foram estatisticamente superiores às espécies com maior valor de importância. Considerando que o ICA tem relação direta com o volume de madeira, somado as características de maior altura comercial de Araucaria angustifolia, equivalente a $80 \%$ da altura total, ratifica-se o seu potencial para o manejo florestal.

De fato, as condições ambientais encontradas na UE I resultaram nos menores valores de ICA e de altura comercial para Araucaria angustifolia, evidenciando que o potencial produtivo do sítio interfere no crescimento primário (altura) e secundário (diâmetro) da espécie. Na UE I, também foi encontrada a menor diferença entre as alturas totais entre os grupos, sendo inferior a $1 \%$.

Entre as UEs, a diferença na altura total dos Grupos 1 e 2 foi, em média, $41 \%$ superior no Grupo 1 . No entanto, as restrições do sítio na UE I, como a baixa profundidade efetiva do solo, limitaram o crescimento em altura, tanto para Araucaria angustifolia quanto para as espécies mais representativas.

Embora não diferindo entre as UEs, as maiores alturas totais foram observadas nos sítios em que Araucaria angustifolia mostrou-se como uma espécie indicadora (UEs III, IV e V), sugerindo que a floresta se encontra em estágio avançado de sucessão. Por outro lado, os maiores ICA para Araucaria angustifolia foram encontrados nas UEs II, III e IV, sendo os sítios onde as propriedades do solo, como a estrutura, textura e profundidade, possivelmente exercem maior influência no crescimento da espécie.

A capacidade de uma espécie se desenvolver e competir é dependente dos componentes bióticos e abióticos, sendo o solo um fator primordial (PRITCHETT;
FISHER, 1987). De acordo com Hoogh (1981), Araucaria angustifolia é uma espécie exigente em relação a qualidade de sítio, com taxas elevadas de crescimento em solos férteis a moderadamente férteis. A maior produtividade da espécie é alcançada nos solos argilosos ou franco-argilosos, profundos e bem drenados (SILVA et al., 2001).

Partindo do pressuposto de que o crescimento da espécie está atrelado as condições edafoclimáticas e ao regime regular de chuvas, infere-se que possivelmente as propriedades do solo estão relacionadas com a capacidade produtiva dos sítios, como a porosidade e a capacidade de retenção de água, as quais são responsáveis por uma considerável parcela do crescimento (HOOGH, 1981). Nesse sentido, as propriedades físicas, químicas e biológicas influenciam no desempenho das funções ambientais do solo e expressam sua qualidade (DORAN; PARKIN, 1994).

Para Coomes et al. (2005), as coníferas normalmente são restritas aos sítios menos produtivos, adaptadas aos solos pouco férteis ou mal drenados, os quais limitam o desenvolvimento das espécies folhosas (DUTRA; STRANZ, 2003). Essas características foram observadas na UE I, sítio com muitas espécies higrófilas e com baixa profundidade, que, embora não tenha diferido estatisticamente das demais UEs, manteve as menores médias para as alturas. O menor porte arbóreo do Grupo 2, representado pelas espécies mais importantes, foi justificado pela elevada densidade de árvores do gênero Siphoneugena, que tem como característica uma altura inferior às demais espécies, porém adaptadas às condições do sítio (CARVALHO, 2003).

\section{CONCLUSÕES}

Araucaria angustifolia apresenta crescimento 
diamétrico superior ao das espécies mais representativas da Floresta com Araucária, o que pode ser atribuído pela capacidade de resiliência dessa espécie.

As maiores alturas de Araucaria angustifolia, somada as suas características favoráveis ao manejo, como a elevada altura comercial, evidenciam seu potencial madeireiro.

Os locais a serem considerados prioritários para o manejo florestal consistem em sítios com estrutura e textura do solo que possam favorecer a drenagem, a efetiva aeração do solo e a retenção de água disponível, bem como profundidade para o desenvolvimento das raízes, sendo estas características das UEs II, III e IV.

\section{REFERÊNCIAS}

BACKES, P.; IRGANG, B. Mata Atlântica: as árvores e a paisagem. Porto Alegre: Paisagem do Sul, 2004. 396 p.

BRASIL. Lei no 11.428, de 22 de dezembro de 2006. Dispõe sobre a utilização e proteção da vegetação nativa do Bioma Mata Atlântica, e dá outras providências. Diário Oficial da União, Brasília, DF, n. 246, 26 dez. 2006. Seção 1, p. 1.

BRASIL. Ministério da Agricultura. Departamento Nacional de Pesquisa Agropecuária. Divisão pedológica. Levantamento de reconhecimento dos solos do Estado do Rio Grande do Sul. Recife, 1973. (DNPEA. Boletim técnico, 30).

CARLUCCI, M. B.; JARENKOW, J. A.; DUARTE, L. S.; PILLAR, V. D. Conservação da Floresta com Araucária no Extremo Sul do Brasil. Natureza \& Conservação, v. 9, n. 1, p. 111-114, 2011.

CARVALHO, P. E. R. Espécies arbóreas brasileiras. v. 1. Colombo: Embrapa Florestas, 2003. 1039 p.

COOMES, D. A.; ALLEN, R. B.; BENTLEY, W. A.; BURROWS, L. E.; CANHAM, C. D.; FAGAN, L.; FORSYTH, D. M.; GAXIOLA-ALCANTAR, A.; PARFITT, R. L.; RUSCOE, W. A.; WARDLE, D. A.; WILSON, D. J.; WRIGHT, E. F. The hare, the tortoise and the crocodile: the ecology of angiosperm dominance, conifer persistence and fern filtering. Journal of Ecology, v. 93, n. 5, p. 918-935, 2005.

DORAN, J. W.; PARKIN, T. B. Defining and assessing soil quality. In: DORAN, J. W.; COLEMAN, D. C.; BEZDICEK, D. F.; STEWART, B. A. (Ed.). Defining soil quality for a sustainable environment. Madison: American Society of Agronomy \& Soil Science Society of America, 1994. p. 03-21.

DUTRA, T. L.; STRANZ, A. História das Araucariaceae: a contribuição dos fósseis para o entendimento das adaptações modernas da família no Hemisfério Sul, com vistas a seu manejo e conservação. In: RONCHI, L. H.; COELHO, O. G. W. (Ed.). Tecnologia diagnóstico e planejamento ambiental. São Leopoldo. UNISINOS, 2003. p. 293-351.

EMBRAPA. Empresa Brasileira de Pesquisa Agropecuária. Centro Nacional de Pesquisa de Solos. Sistema brasileiro de classificação de solos. 3. ed. Brasília: Embrapa, 2013. 353 p.
FIGUEIREDO FILHO, A.; DIAS, A. N.; STEPKA, T. F.; SAWCZUK, A. R. Crescimento, mortalidade, ingresso e distribuição diamétrica em Floresta Ombrófila Mista. Floresta, v. 40, n. 4, p. 763-776, 2010.

FIGUEIREDO FILHO, A.; HUBIE, S. R.; SCHAAF, L. B.; FIGUEIREDO, D. J.; SANQUETTA, C. R. Avaliação do incremento em diâmetro com o uso de cintas dendrométricas em algumas espécies de uma Floresta Ombrófila Mista localizada no Sul do Estado do Paraná. Revista Ciências Exatas e Naturais, v. 5, n. 1, p. 69-84, 2003.

HOOGH, R. J. Site-nutrition-growth relationships of Araucaria angustifolia (Bert.) O. Ktze. in southern Brazil. $161 \mathrm{f}$. 1981. Tese (Doutorado em Silvicultura) - Universidade de Freiburg, Freiburg, 1981.

IRIARTE, J.; BEHLING, H. The expansion of Araucaria forest in the southern Brazilian highlands during the last 4000 years and its implications for the development of the Taquara/Itararé Tradition. Environmental Archaeology, v. 12, n. 2, p. 115-127, 2007.

$\mathrm{KOCH}$, Z.; CORRÊA, M. C. Araucária: a floresta do Brasil Meridional. 2. ed. Curitiba: Olhar Brasileiro, 2010. 168 p.

LEITE, P. F.; KLEIN, R. M. Vegetação. In: IBGE. Instituto Brasileiro de Geografia e Estatística (Ed.). Geografia do Brasil: Região Sul. Rio de Janeiro: IBGE, 1990. p. 113-150.

MALUF, J. R. T. Nova classificação climática do Estado do Rio Grande do Sul. Revista Brasileira de Agrometeorologia, v. 8, n. 1, p. 141-150, 2000.

MATTOS, P. P.; SANTOS, A. T.; RIVERA, H. OLIVEIRA, Y. M. M. de; ROSOT, M. A. D.; GARRASTAZU, M. C. Crescimento de Araucaria angustifolia na reserva florestal Embrapa / Caçador, SC. Pesquisa Florestal Brasileira, n. 55, p. 107-114, 2007.

MORENO, J. A. Clima do Rio Grande do Sul. Porto Alegre: Secretaria da Agricultura, 1961. 42 p.

MYERS, N.; MITTERMEIER, R. A.; MITTERMEIER, C. G.; FONSECA, G.A. B.; KENT, J. Biodiversity hotspots for conservation priorities. Nature, v. 403, p. 853-858, 2000.

PRITCHETT, W. L.; FISHER, R. F. Properties and management of forest soils. 2. ed. New York: John Wiley \& Sons, 1987. 494 p.

RODERJAN, C. V.; GALVÃO, F.; KUNIYOSHI, Y. S.; HATSCHBACH, G. G. As unidades fitogeográficas do estado do Paraná. Ciência \& Ambiente, n. 24, p. 75-92, 2002.

SCHÄFFER, W. B.; PROCHNOW, M. Mata Atlântica. In: SCHÄFFER, W. B.; PROCHNOW, M. (Ed.). A Mata Atlântica e você: como preservar, recuperar e se beneficiar da mais ameaçada floresta brasileira. Brasília: APREMAVI, 2002. p. 12-44.

SILVA, H. D.; BELLOTE, A. F. J.; FERREIRA, C. A.; BOGNOLA, I. A. Recomendação de solos para Araucaria angustifolia com base nas suas propriedades físicas e químicas. Boletim Pesquisa Florestal, n. 43, p. 61-74, 2001. 